\title{
Integrating Electrical Analogy and Computer Modeling of Groundwater Flow for Teaching Flownet Concepts
}

\author{
http://dx.doi.org/10.3991/ijep.v3i4.3052
}

\author{
M. Kasi ${ }^{1}$, Y. Chi ${ }^{1}$ and G. Padmanabhan ${ }^{2}$ \\ ${ }^{1}$ Moore Engineering, Inc., West Fargo, USA \\ ${ }^{2}$ North Dakota State University, Fargo, USA
}

\begin{abstract}
Teaching complex engineering problems can often be enhanced by utilizing students' prior knowledge in analogously similar problems. Flow of electrical current through a conducting medium is analogous to groundwater flow through a porous medium because both are governed by Laplace equation. This analogy was used in an alternate representational approach by the authors in teaching flownet concepts. The authors employed the easily demonstrable electrical counterpart to represent the groundwater flow problem in a laboratory setting. This paper discusses the efforts of authors to teach the flownet principles better by using the electrical analogy for selected groundwater flow situations. However, it was also demonstrated that the electrical analogy concept can be applied for a wide range of groundwater flow situations with a few simple modifications. Students also used a groundwater flow computer model to obtain flownets for the same flow situations as the ones that were obtained using an electrical analogy. The student feedback indicated that this approach could improve student learning of flownet concepts.
\end{abstract}

Index Terms-Electrical analogy, flownet, groundwater, laboratory module, Visual MODFLOW.

\section{BACKGROUND}

Flownets are convenient graphical representations of steady patterns of groundwater flow consisting of equipotential lines and stream or flow lines. Equipotential lines are lines along which a constant potential exists. Flow lines are lines along which the velocity vectors are tangents. By definition, flow lines and equipotential lines must be orthogonal to one another. In order to simplify flow calculations, these two families of curves are usually drawn to form a pattern of curvilinear squares. Flownets can be constructed by trial and error graphically or by using the governing equations with appropriate boundary conditions. The flownet concept is applicable to all steady-state flow situations in which the flow of any flux is driven by a potential difference and thereby the governing equation is a LaPlace equation. For instance, groundwater flow, flow of heat in a heat-conducting medium, flow of magnetic flux, and flow of current in an electrical conducting medium, are all governed by the Laplace equation and flownet concepts are applicable to all these situations. The LaPlace equation in a two-dimensional form is given as follows:

$$
\frac{\partial^{2} \phi}{\partial x^{2}}+\frac{\partial^{2} \phi}{\partial y^{2}}=0
$$

where ' $\phi$ ' is the potential. However, the potential $\phi$ in different disciplines is different. For instance, $\phi$ may refer to the hydraulic potential in flow through porous media (soil), to the temperature in heat conduction, or to the voltage in a current conduction. The basic common feature (analogy) of all these phenomena enables one to transfer the solution of a problem in one discipline to a similar problem in another discipline. In this experiment we will obtain the flownet pattern for a groundwater flow situation using the electrical counterpart of the problem. Appropriate geometry and boundary conditions must be applied to obtain solutions of specific problems.

The construction of a flownet is an indirect way of obtaining the solution to a LaPlace equation with appropriate boundary conditions [1]. This property of a flownet makes it possible to solve a problem in one discipline as an analogous problem in another discipline having the LaPlace equation as the governing equation. Hence, concepts of flownets can be employed to obtain solutions of a steady-state groundwater flow problem by obtaining flownets of an analogous electrical current flow problem. A simple analogy between groundwater flow and electrical flow is presented in Table 1.

TABLE I.

THE ANALOGY

\begin{tabular}{|c|c|}
\hline Groundwater Flow & Electrical Current Flow \\
\hline$\frac{\partial^{2} h}{\partial \mathrm{x}^{2}}+\frac{\partial^{2} \mathrm{~h}}{\partial \mathrm{y}^{2}}=0$ & $\frac{\partial^{2} \mathrm{v}}{\partial \mathrm{x}^{2}}+\frac{\partial^{2} \mathrm{v}}{\partial \mathrm{y}^{2}}=0$ \\
\hline Darcy's Law: $\mathrm{V}_{\mathrm{x}}=-\mathrm{K} \frac{\partial \mathrm{y}}{\partial \mathrm{x}}$ & Ohm's Law: $\mathrm{I}_{\mathrm{x}}=-\sigma \frac{\partial \mathrm{v}}{\partial \mathrm{x}}$ \\
\hline
\end{tabular}

where $V_{x}$ is velocity in the $x$-direction (discharge/area), $I_{x}$ is electrical conductivity, $\mathrm{K}$ is hydraulic conductivity, $\sigma$ is specific electrical conductivity, $\mathrm{h}$ is hydraulic potential, and $\mathrm{v}$ is electrical potential (voltage).

Obtaining flownets for groundwater flow problems using this method is not new. Past studies used electrical analogy to obtain groundwater flow patterns [2], hydraulic properties of aquifers [3], and contaminant transport [4]. The authors have taught the flownet principles using this analogy in the past, but without computer models in a laboratory setting [5]. However, this method has not been widely used for teaching in a laboratory setting by integrating groundwater flow principles, its electrical analogy, and groundwater flow computer models. Flownets of groundwater flow can also be obtained by using computer models. Visual MODFLOW is a popular groundwater flow model which simulates groundwater flow and con- 
taminant transport through a porous medium using a block-centered finite-difference method [6]. The model can simulate common boundary conditions generally encountered in practice.

The impact of "representations" approach on learning has been studied extensively in educational psychology [7, 8]. The role of representational fluency in the development of deeper and higher-order understandings of important concepts in engineering education has been researched by several investigators [9, 10, 11, and 12]. Felder et al., [10] emphasize the need to put students into learning environments that provide them with opportunities to think with and through multiple representations. Developing and using representations is an essential skill that engineering students need to learn. Representational fluency is a documented measure to assess student understanding of engineering concepts [13]. Thinking about a problem through multiple representations and through translations within and among representations contributes to conceptual and application understanding of undergraduate students [14].

In this paper, we present our experience with an instructional unit as part of our civil engineering undergraduate Fluid Mechanics laboratory for exploring flownet concepts in groundwater flow through an electrical circuit analogy and through the use of a computer model, Visual MODFLOW. Electrical analogy is an alternate representation of groundwater flow which allows students to relate the groundwater flow problem to their prior knowledge of electrical current concepts. On the other hand, Visual MODFLOW allows a visual representation of groundwater flow problem. However, students often have difficulties understanding newly introduced concepts, such as groundwater flow through soils with different hydraulic conductivities. An instruction combining electrical analogy (an alternate representation) and Visual MODFLOW (a visual but abstract representation) in such situation is often helpful for the students to quickly understand new concepts $[15,16]$.

Five different practical groundwater flow situations were modeled (represented) through corresponding analogous circuit set-ups for instruction. First, basic concepts of groundwater flow, electrical analogy, flownets, and the Visual MODFLOW software features were taught. Then the students were asked to develop flownets using the electrical analogy experiments and compare the results with their Visual MODFLOW simulations. Student feedback was obtained to understand the effectiveness of the approach with regard to improving student learning.

\section{LABORATORY EXPERIMENT}

The flownet experiment was included as one of the twelve experiments in the Fluid Mechanics Laboratory course (CE 310), a 1-credit required course for Civil Engineering in the undergraduate curriculum [17]. The objective of the experiment is to impart to the students the following concepts and enable them to understand and interpret flownets:

- Hydraulic head and groundwater velocity

- Darcy's Law

- Hydraulic conductivity and electrical conductivity

- LaPlace equation
- Boundary conditions (constant head, permeable, and impermeable)

- Electrical analogy to groundwater flow

- Solution of LaPlace equation for groundwater flow

- Solution of LaPlace equation for current flow

- Contours of hydraulic head/voltage

- Equipotential lines, flow lines, and flownet

- Interpretations of flownets

- Groundwater flow computer software

\section{A. Experimental Setup}

The electrical analogy experiment setup consisted of a plexiglass tray (24 inches long, 24 inches wide, and 4 inches deep), electrodes (aluminum sheets), a step-down transformer, a voltmeter, a voltage probe, and a graphing paper (Fig. 1). Additionally, a wooden block (6 inches long, 2 inches wide, and 2 inches deep), an aluminum cylinder ( 2 inches of external diameter and 4 inches long), and a steel cylinder (4 inches of external diameter) were used in setting up the electrical counterparts of the different groundwater flow situations. Materials used in the electrical analogy setup are presented in Fig. 1. The tray was filled with tap water to form a thin sheet of water $(<1$ inch) to serve as the medium to conduct electricity. The tap water is analogous to soil, which is the conducting medium for ground water flow. Electrodes were placed on the tray at suitable locations depending on the flow situation to be simulated.

Voltage at any point on the conducting medium of water could be measured using the voltmeter and the probe. A graphing paper was placed underneath the tray to facilitate locating coordinate points of constant voltage readings and to plot constant voltage lines (equipotential lines). Curvilinear stream lines were drawn perpendicular to these equipotential lines to construct flownets. Electrical supply can be provided through direct current (DC) using electric storage batteries or alternating current (AC) using a step-down transformer. Electrical power supply to the electrodes in the present teaching module was provided through a step-down transformer, which was used to bring the voltage down from $120 \mathrm{~V}$ to $20 \mathrm{~V}$.

\section{B. Selected Groundwater Flow Situations}

Students were presented with five different groundwater flow situations and were asked to

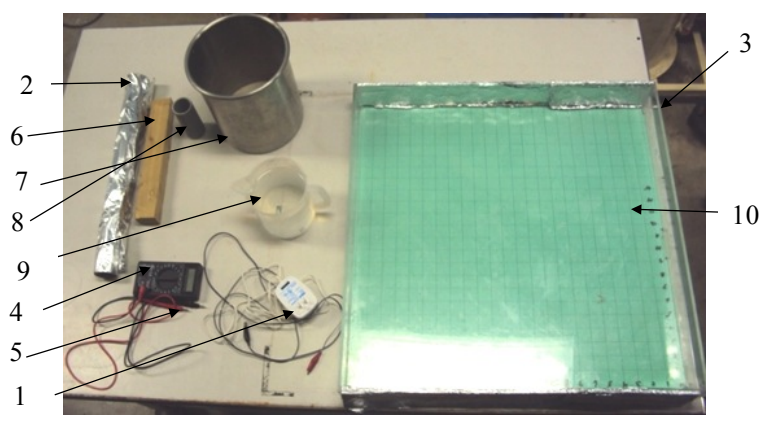

Figure 1. Materials used in the electrical analogy setup to develop flownets for various groundwater flow situations. 1 - step-down transformer, 2 - aluminum sheet, 3 - plexiglass tank, 4 - voltage meter, 5 probe, 6 - wooden block, 7 - steel cylinder, 8 - aluminum cylinder, 9 tap water, and 10 - graphing paper. 
conceptualize the analogous problem, prepare the corresponding electrical analogy setup, and take measurements of spatial distribution of voltage on the conducting medium (water in the tray) to obtain flownets. Also, students were asked to simulate the flow situations in Visual MODFLOW and compare the patterns obtained from electrical analogy. The five selected flow situations with increasing complexity are:

1. Groundwater flow between two constant head boundaries (Fig. 2a and 2b)

2. Groundwater flow between two constant head boundaries with an "impermeable barrier" in between the boundaries (Fig. 2c)

3. Groundwater flow between two constant head boundaries with a "permeable barrier" in between the boundaries (Fig. 2d)

4. Groundwater flow converging to a pumping well situated near a recharge boundary (Fig. 2e)

5. Seepage beneath a dam (Fig. 2f)

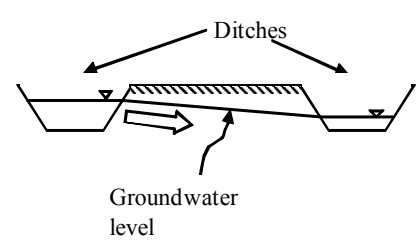

(a)

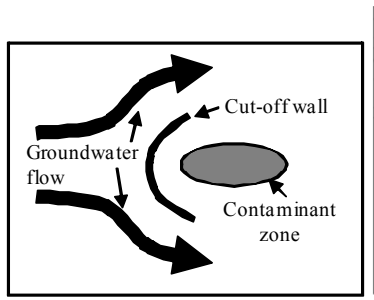

(c)

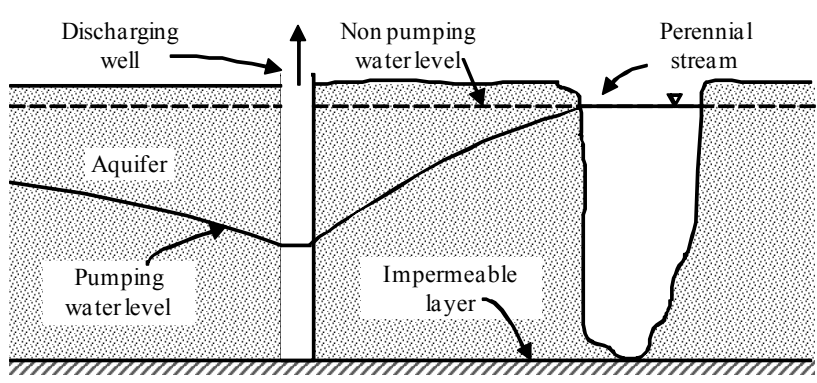

(e)

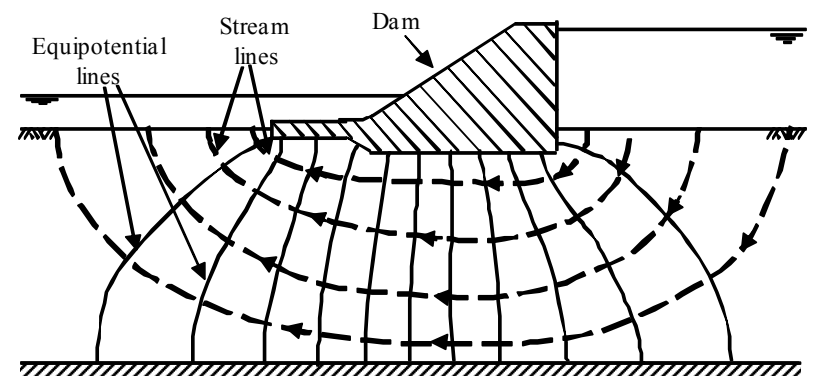

(f)

Figure 2. Groundwater flow situations: (a) between two ditches through agricultural fields, (b) Seepage from a pond, (c) around an impermeable barrier, (d) through a permeable barrier, (e) near a pumping well, (f) underneath a dam.
In the first situation, the groundwater flow through a homogeneous soil between two constant head boundaries was simulated. This situation could be an example for groundwater flowing between two lakes at different elevations or two ditches at different elevations in an agricultural field (Fig. 2a) or infiltration of water from the bottom of a pond to an unconfined aquifer (Fig. 2b).

While keeping the constant head boundaries equal, an impermeable barrier was added in between the two boundaries in the second situation. This situation represents the grout/slurry walls (or cut-off walls) that divert the groundwater flow from a contaminated zone (Fig. 2c). Vertical barrier walls are often used for waste containment by diverting the flow of uncontaminated groundwater from passing through a contaminated site [18, 19]. The walls could be typically straight, curved, or enclosed, and constructed of grout or clay slurry with plastic or steel sheet piling.

In the third situation, the impermeable barrier was replaced by a semi-permeable barrier. This situation simulates the flow in a permeable reactive barrier (PRB) system (Fig. 2d), which is an in situ remediation technology to treat contaminated ground water. The PRB system combines a passive chemical or biological treatment zone with subsurface groundwater flow management $[20,21]$. One of the typical configurations of PRBs include a reaction vessel which routes groundwater via natural or engineered preferential pathways to a subsurface reaction vessel, where the contaminated water is treated using chemical or biological reactive materials.

The fourth situation simulated a pumping well condition. Two cases were considered in this situation: (1) the effect of a pumping well situated near a perennial stream and (2) the effect of a pumping well located in between two perennial streams. Figure 2e illustrates Case 1 of this situation. The fifth situation simulated seepage underneath a dam (Fig. 2f). The dam was analogously simulated with two cases: 1) homogeneous soil below the dam and 2) an impervious soil layer underneath the dam.

\section{Electrical Analogy Setup of Groundwater Flow Situations}

The constant head boundaries in the first three situations and the fifth situation were simulated by applying a higher voltage to one aluminum sheet and a lower voltage to the other. In the fourth situation, lower voltage was applied to an aluminum cylinder to simulate a pumping well condition. The coordinates of constant voltage points were identified on a graph sheet placed below the plexiglass tank. The situations were developed with straight and/or rectangular edge boundaries. However, with a few modifications, the setup can be used to simulate irregular boundary conditions. The electrical analogy setup for different scenarios is illustrated in Fig. 3.

Two cases were studied for the first situation representing examples presented in Fig. $3 \mathrm{a}$ and $3 \mathrm{~b}$. In Case 1 (Fig. $3 \mathrm{a}$ ), electrodes (aluminum sheets) were placed on the opposite ends of the plexiglass tray. In Case 2 (Fig. 3a), the tray walls were covered on three sides with connecting aluminum sheets to make the three sides as one electrode. The fourth wall of the tray was covered with another aluminum sheet with small gaps between this fourth side and its adjacent sides. A voltage difference of $21.2 \mathrm{~V}$ was maintained between the two electrodes. 
For the second situation, the setup described in Case 1 of the first situation, was slightly modified with the introduction of a wooden rectangular block at the middle of the plexiglass tank as shown in Fig. 3b. The wooden block was used as a material impermeable to electrical current, which is analogous to an impermeable grout wall in the case of groundwater flow. To simulate the third situation, i.e. PRB with reaction vessel, the wooden block was replaced by a steel cylinder. In the present experiments, the steel cylinder which has a higher electrical conductivity than the surrounding water would analogously represent the reaction vessel. The electrical analogy setup for this flow situation is illustrated in Fig. 3c.

The electrical analogy setups for the two cases in the fourth situation are shown in Fig. 3d. An aluminum cylinder was used as analogous to the pumping well and an aluminum sheet on one side of the plexiglass tank to simulate Case 1 and aluminum sheets on opposite sides of the plexiglass tank to simulate Case 2. In Case 1, a voltage difference of $21.2 \mathrm{~V}$ was applied between the aluminum sheet and the aluminum cylinder to simulate the pumping well at the aluminum cylinder. To simulate Case 2, two step-down transformers were used. A voltage difference of 21.2 $\mathrm{V}$ between one of the aluminum sheets and the aluminum cylinder was created with one step-down transformer. Using the second transformer, a voltage difference of $13 \mathrm{~V}$ was created at the second aluminum sheet by earthing one of the electric circuit ends.

The electrical analogy for the fifth situation was set up as shown in Figure 3e. Aluminum sheets were placed on the same side of the plexiglass tray with a gap in between them (Case 1 in Fig. 3e). The gap which is electrically resistant is analogous to the dam. A voltage difference of 21.2 V was maintained between the two electrodes. To simulate the impervious soil layer in Case 2, polyethylene foam was cut into an irregular shape and glued to the plexiglass tank. Polyethylene foam which is an electrically resistant material is analogous to the impervious soil layer.

\section{ApplicAtion of "Visual ModFlow"}

The flow area was discretized into 20 rows and 20 columns with equal size square cells for the Visual MODFLOW simulations. The flow zone was conceptualized as unconfined, single layer, homogeneous as well as isotropic and the flow as steady-state. Boundary conditions were defined using the boundary module in Visual MODFLOW according to the conditions described in each groundwater flow situation in the following paragraphs. Visual MODFLOW's "constant head boundary" module fixes the head value in selected grid cells, i.e. there is no change with time, it thus acts as an infinite source of water entering the system or as an infinite sink for water leaving the system depending on the water level in the surrounding cells. All situations were simulated in a steady-state analysis.

Thus, similar to the setup shown for both cases ( 1 and 2) of the first situation in Fig. 3(a), the boundaries in Visual MODFLOW were defined as constant head boundaries with head values of $21.2 \mathrm{ft}$ and $0 \mathrm{ft}$, respectively. For the second situation, the impermeable barrier was simulated by an independent module called "Wall" Boundary or Horizontal Flow Barrier (HFB) package in the Visual MODFLOW. The HFB package requires input of two parameters from the user: thickness and conductivity. A (a)

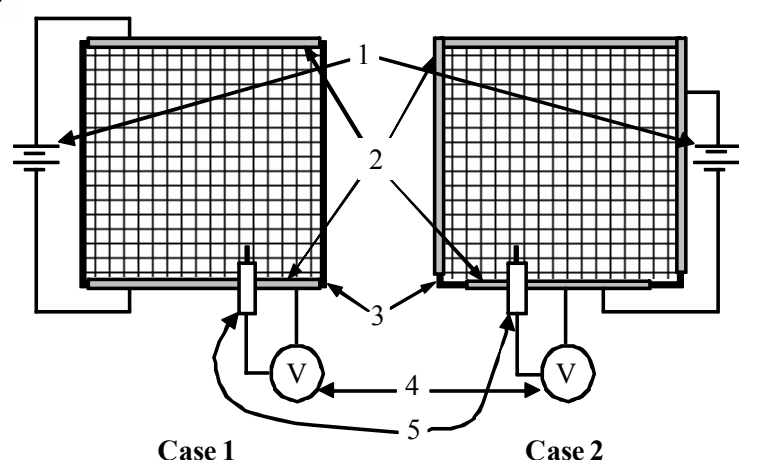

(b)

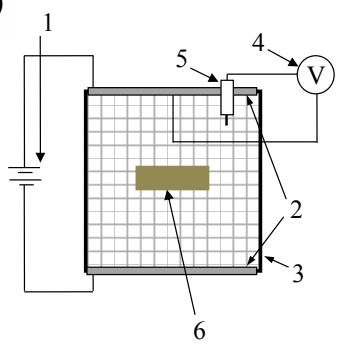

(c)

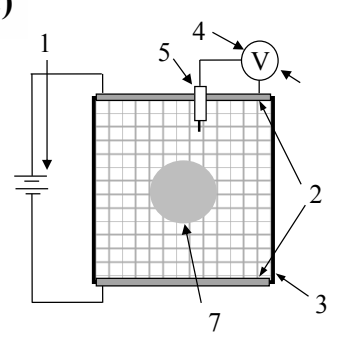

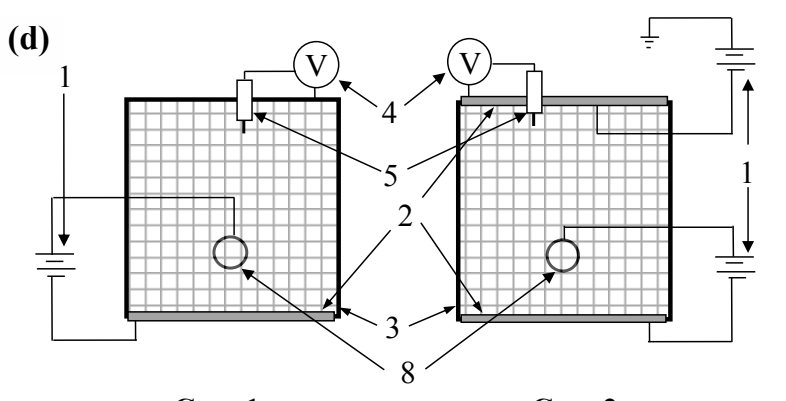

Case 1

Case 2

(e)

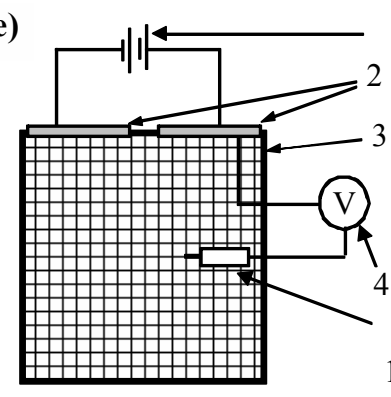

Case 1

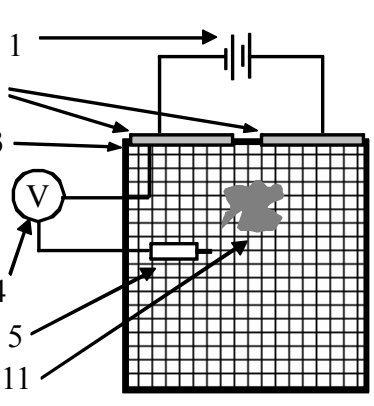

Case 2
Figure 3. Electrical analogy setup. (a) situation 1, (b) situation 2, (c) situation 3, (d) Situation 4 and (e) Situation 5.1 - step-down transformer, 2 - aluminum sheet, 3 - plexiglass tank, 4 - voltage meter, 5 - probe, 6 - wooden block, 7 - steel cylinder, 8 - aluminum cylinder, 9 - tap water, 10 - graphing paper, and 11- styrofoam

horizontal thickness of $1 \mathrm{ft}$ and a conductivity of 0.005 $\mathrm{ft} /$ day were used in the model for this scenario. For the third situation, a higher hydraulic conductivity value was assigned to the cells where PRB is located than the surrounding cells (100 $\mathrm{ft} /$ day versus $3 \mathrm{ft} /$ day $)$. Visual MODFLOW's "Pumping well" module was used to simulate the fourth situation. One constant head boundary of $21.2 \mathrm{ft}$ was used in Case 1, while two constant head boundaries of $21.2 \mathrm{ft}$ and $13 \mathrm{ft}$ were used in Case 2. A pumping rate of $30,000 \mathrm{ft}^{3} /$ day was assigned to the well in both cases. For the fifth situation, constant head bounda- 
ries of $21.2 \mathrm{ft}$ and $0 \mathrm{ft}$ were defined on the same side of the aquifer with a few no-flow cells in between these constant head boundaries. These no-flow cells represent the dam condition. In Case 2 of this situation, a hydraulic conductivity of $1 \times 10^{-6} \mathrm{ft} /$ day was assigned to cells in the impervious soil layer region as compared to $10 \mathrm{ft} /$ day for the surrounding cells.

\section{STUDENT FEEDBACK}

A short survey consisting of four questions (A-D below) was given to the students in order to get their feedback on the flownets exercise.

A. Did the electrical analogy experiment help you understand better the

1. concept of flownet?

2. idea of equipotential lines and flowlines and their relationship?

3. analogy between the flow of groundwater and the flow of current through a conducting medium?

4. patterns of groundwater flow situations?

B. Which part of the experiment did you like the most?

C. What could be done to improve and enhance the experiment?

D. Are there any other groundwater flow scenarios that you would like to observe with this electrical analogy experiment?

Question A consisted of four sub-parts designed to elicit Yes/No type answers. The purpose of these questions was to assess whether the students understood the concept of flownets and patterns of groundwater flow under different boundary conditions from electrical analogy experiments. Questions B through D were short-answer, openended questions, asking the students to identify the part of the experiment they liked most, to suggest improvements and enhancements to the experiment, and to describe any additional scenarios that they would like to observe with the electrical analogy experiment.

\section{EXPERIMENT RESULTS}

The results obtained from the students regarding both electrical analogy experiments and the Visual MODFLOW simulations for selected scenarios are presented in Fig. 4. In the Visual MODFLOW simulations, the parameters of aquifer properties and/or pumping rate were adjusted to get comparable definitions of flownets. Instructors provided the necessary guidance to the students in selecting the values for different model parameters. The results for the three selected scenarios $(2,4-2$, and 5-1) for electrical analogy are presented in Fig. 4 (a), (c) and (e), and their corresponding results concerning Visual MODFLOW are presented in Fig. 4 (b), (d) and (f).

Flownet patterns from electrical analogy and Visual MODFLOW closely agreed with each other. Flownets constructed for groundwater flow through a permeable barrier are presented in Fig. 4 (a) and (b). The equipotential lines bent away from the barrier for a permeable barrier and the flow lines were bent into the barrier. Due to the higher conducting nature of the barrier (steel cylinder) than the surrounding medium (mildly ionized water), the
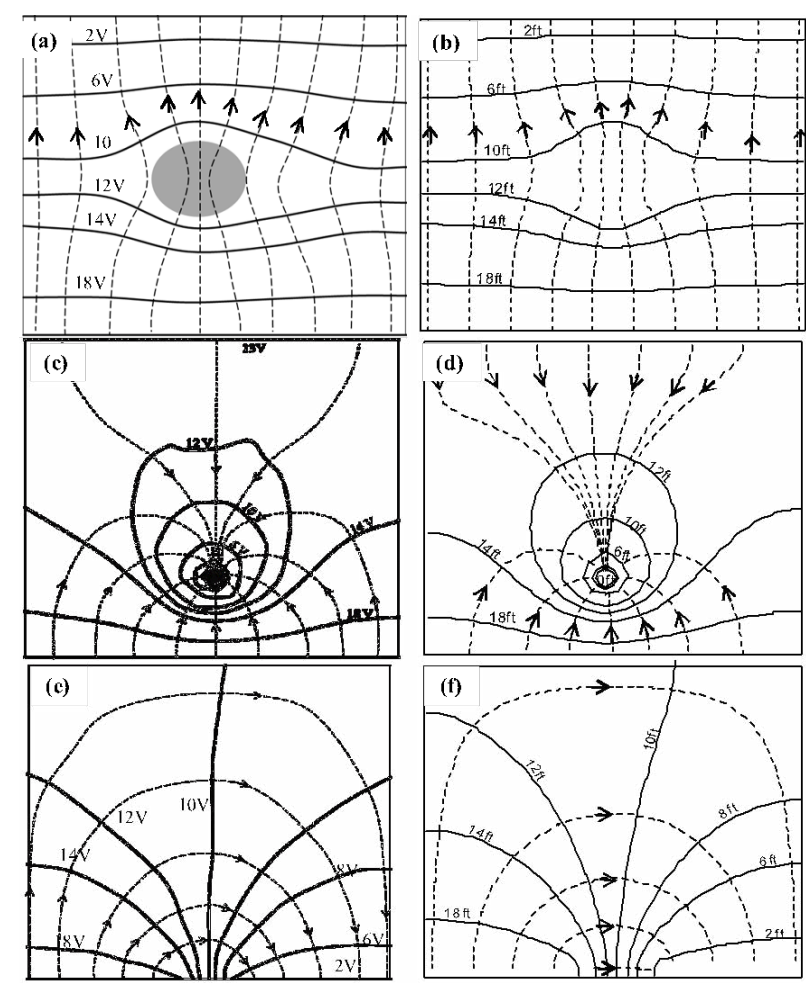

Figure 4. Flownets obtained from electrical analogy (a, c and e) and Visual MODFLOW (b, $\mathrm{d}$ and $\mathrm{f}$ ) simulations for situations 2, 4-2, and 51.

flow lines (current) have a tendency to pass through the highly-permeable material.

The flownets results for a pumping well with two constant head boundaries are presented in Fig. 4 (c) and (d). The flowlines are closer to each other near the $21.2 \mathrm{~V}$ (21.2 ft in Visual MODFLOW result) boundary than next to the $13 \mathrm{~V}$ boundary. Since the pumping well is close to the constant head boundary of $21.2 \mathrm{~V}$, the second boundary in Case 2 has a minor effect on the groundwater levels in the aquifer. The flownets clearly indicate the formation of a depression cone due to the intense pumping rate drawing water from the aquifer around the pumping well within a certain distance.

The flownets presented in Fig. 4 (e) and (f) illustrate the groundwater flow pattern beneath a dam. Close to the dam, the flow path lines are shorter. The equipotential lines are closer together right below the dam indicating higher potential gradients and hence higher velocities than those further away from the dam.

\section{StUdent FEEDBACK Results}

A total of 48 students responded to the experiment in the Spring semester of 2010. The responses to Question A are shown in Fig. 5. Almost all the students (more than $95 \%$ ) indicated that they gained a better understanding of flownet concepts. Individual descriptive responses to Questions B-D are summarized below in Table 2. Student responses to Questions B and C were grouped under different categories and the frequency for each group was calculated. One representative response from each group is presented in Table 2. A total of 34 students responded to Question B while 18 responded to Question C. Distinct groups of five and four were identified for Questions B and $\mathrm{C}$ and the frequency of responses for each group is summarized in Fig. 6 and 7. The maximum number of 
responses (35\% of the total responses) for Question B indicated "observational learning" as the enjoyable part of the experiment. The students also felt that there was a need for more guidance during the experiments $(35 \%$ of total responses). Personal communication with the students indicated that the additional guidance was especially sought during the electrical analogy setup for complex groundwater flow situations (for example, Scenario 4 Case 2). In general, the responses indicate that the students have enjoyed conducting experiments on the different scenarios chosen and were able to visualize the groundwater flow situations through analogous comparisons.

The majority of students did not mention any improvements over existing experiments or the experimental setup (responses to Question C). A few students felt that more readings at closer intervals would have given them a better picture of groundwater flow patterns in complex scenarios such as flow around a barrier. This was included as a recommendation to the teaching assistants in future semesters. Moreover, students also wanted to explore a few other scenarios such as "Beaver Dam" and "infiltration and inflow into sewer pipes". These responses show that the analogy experiment has stimulated the critical and analytical thinking of the students.

\section{INSTRUCTOR REFLECTIONS AND RECOMMENDATIONS}

The Fluid Mechanics laboratory course is often taught by multiple instructors in different sections, which brings about a variation in the delivery of materials. For wellestablished lesson plans, the variation is gradually minimized by following specific protocols in the teaching modules. Since the electrical analogy concept was newly introduced, one instructor was made available to all the sections during both the semesters to bring uniformity in the delivery of instruction. The second important element during this session is the active involvement of all the students in conducting the experiments. This active involvement was necessary to ensure that the students clearly understand the methods of taking equipotential points on the graphing paper, of making adjustments for various scenarios, and of analyzing the results during the development of flownets. The instructors needed to be proactive in constantly verifying the reasons behind each step in the electrical analogy model setup, linking each step in the setup with real world conditions during the experiments, and providing instructions on the methods used to draw flownets. Above all, an interactive discussion with the instructors based on the reports submitted by the students was necessary to ensure that students understood the concepts thoroughly.

A few modifications in the evaluation process could be considered for getting student feedback in the future when offering this laboratory unit. Questions A.1 to A.4 may be modified from yes/no type to a Likert scale to obtain a finer-grained evaluation. In addition, a control-based approach can be taken, whereby the progress of one group of students through the groundwater flow experiment with electrical analogies can be measured. This shall be compared with another group without the analogies as a better method of assessing the improvement of student learning. The students' knowledge and perceptions among the two groups could be compared to evaluate the effectiveness of the method. Although the authors would have preferred

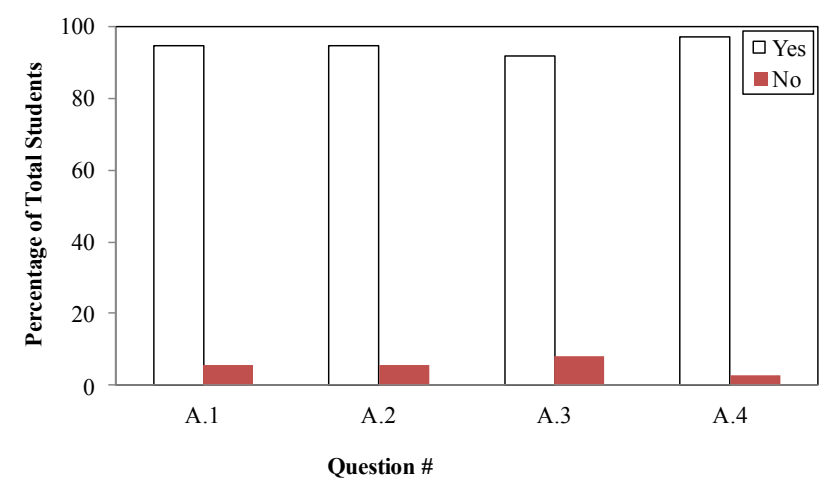

Figure 5. Student feedback on electrical analogy experiments.

TABLE II.

RESPONSES TO SURVEY QUESTIONS B, C, \& D

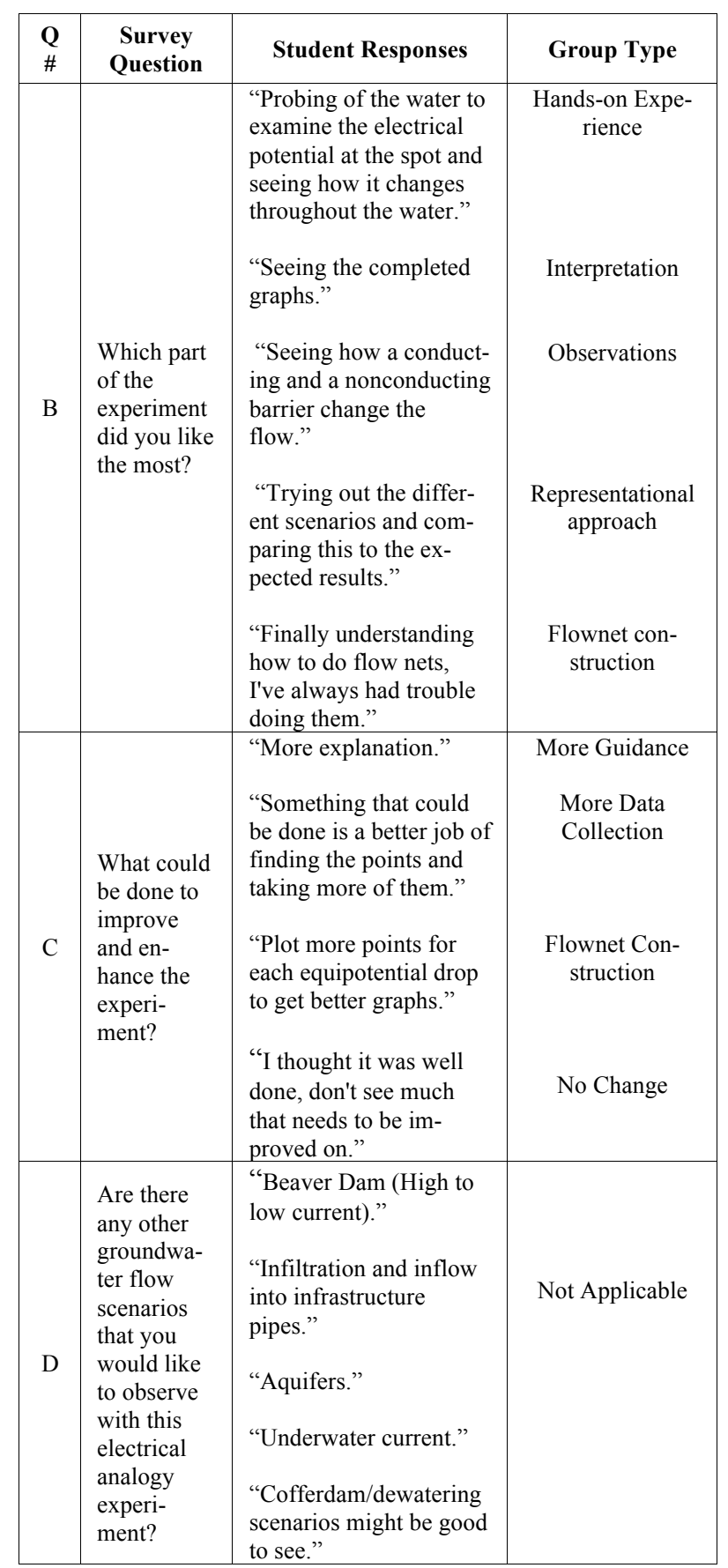




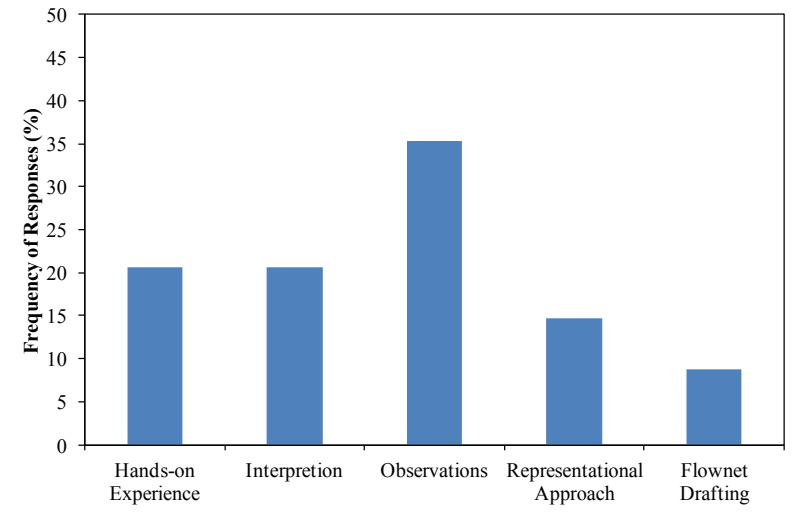

Figure 6. Group-wise frequency of responses for Question B.

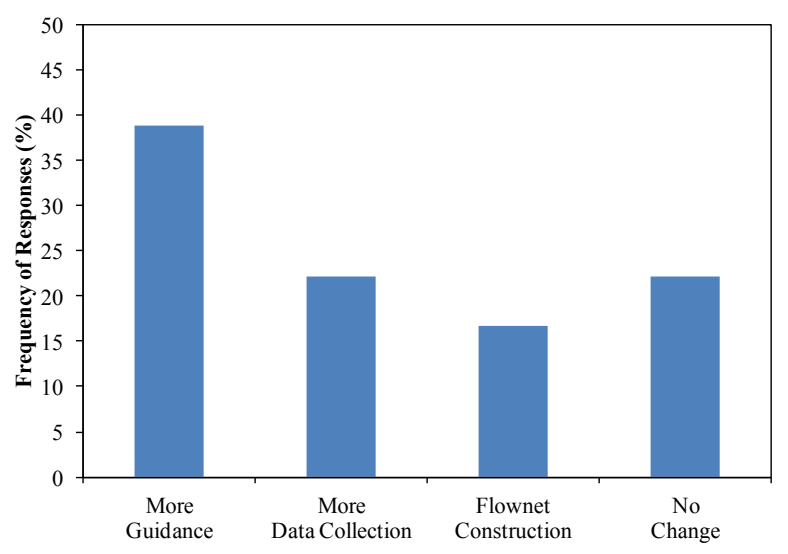

Figure 7. Group-wise frequency of responses for Question C.

this control-based approach, it was not feasible to implement under the available set up. Besides, the electrical analogy part is the only experimental part and the unit would not exist without it.

\section{CONCLUSIONS}

The different concepts of bringing flownets into the lab were introduced to the undergraduate civil engineering students in a laboratory course by integrating the principles of groundwater flow, of the electrical analogy of groundwater flow, and of the use of a groundwater flow computer model. An experiment was developed and implemented to illustrate flownets of different carefully selected groundwater flow situations using their analogous problems of current flow through an electrically conducting medium. The setup was introduced with simple geometric boundaries, however, it was also demonstrated that with a few easy modifications, it can be applied to groundwater flow situations with irregular boundary conditions, too. The students obtained results from the electrical analogy experiments that were comparable to those from the groundwater flow modeling software, Visual MODFLOW. The assessment based on student evaluations at the end of the experiment indicated that the students understood the basic idea behind the application of electrical analogy for various groundwater flow situations and the concept of flownets. The integration of the electrical analogy of ground water flow and the use of a computer model to explore the concepts of flownets in a laboratory setting has proved to be unique and appealing to the students.

\section{ACKNOWLEDGMENTS}

The authors would like to thank the 2010 Spring and Fall semester teaching assistants of the Fluid Mechanics laboratory, Department of Civil Engineering, North Dakota State University for their assistance to the students when conducting the experiments.

\section{REFERENCES}

[1] H. R. Cedergren, Seepage, Drainage and Flownets. 3rd ed., New York: John Wiley \& Sons, Inc., 1989.

[2] M. S. Bedinger, "An Electrical Analog Study of the Geometry of Limestone Solution," Ground Water, vol. 5, no. 1, pp.24-28, 1967. http://dx.doi.org/10.1111/j.1745-6584.1967.tb01235.x

[3] C. C. Ezeh, "Geoelectrical Studies for Estimating Aquifer Hydraulic Properties in Enugu State, Nigeria,” Int. J. Phys. Sci., vol. 6, no. 14, pp.3319-3329, 2011.

[4] J. L. Osiensky, and P. R. Donaldson, "Electrical Flow Through an Aquifer for Contaminant Source Leak Detection and Delineation of Plume Evolution," J. Hydrol., vol. 169, no. 1-4, pp.243-263, 1995. http://dx.doi.org/10.1016/0022-1694(94)02610-N

[5] M. Kasi, Y. Chi, and G. Padmanabhan, "Teaching Flownet Concepts to Engineering Undergraduates Using Electrical Analogy of Groundwater," AC 2011-84, Proceedings of the $118^{\text {th }}$ Annual Conference \& Exposition, ASEE, June 26-29, 2011, Vancouver, Canada.

[6] Schlumberger Water Services, User's Manual 2010.1 Visual MODFLOW, Dynamic Groundwater Flow and Contaminant Transport Modeling Software, 2010.

[7] F. M. Dwyer, "The Effectiveness of Visual Illustrations Used to Complement Programmed Instruction," Journal of Psychology, vol. 70, no. 2, pp. 157-162, 1968. http://dx.doi.org/10.1080/00223980.1968.10544944

[8] K. R. Butcher, "Learning from Text with Diagrams: Promoting Mental Model Development and Inference Generation," Journal of Educational Psychology, vol. 98, no. 1, pp. 182-197, 2006. http://dx.doi.org/10.1037/0022-0663.98.1.182

[9] R. Felder, and L. K. Silverman, "Learning and Teaching Styles in Engineering Education," Engineering Education, vol. 78, no. 7, pp. 674-681, 1988.

[10] R. Felder, D. Woods, J. Stice, and A. Rugarcia, "The Future of Engineering Education. II. Teaching Methods That Work," Chemical Engineering Education, vol. 34, no.1, pp. 26-39, 2000.

[11] J. Moss, K. Kotovsky, and J. Cagan, "The Role of Functionality in the Mental Representations of Engineering Students: Some Differences in the Early Stages of Expertise," Cognitive Science, vol. 30 , no. 1, pp. 65-93, 2006. http://dx.doi.org/10.1207/ $\underline{\mathrm{s} 15516709 \operatorname{cog} 0000 \quad 45}$

[12] R. A. Streveler, T. A. Litzinger, R. L. Miller, and P. S., Steif, "Learning Conceptual Knowledge in the Engineering Sciences: Overview and Future Research Directions," Journal of Engineering Education, vol. 97, no. 3, pp. 279-294, 2008. http://dx.doi.org/10.1002/j.2168-9830.2008.tb00979.x

[13] A. Johri, and V. Lohani, "A Framework for Improving Engineering Representational Literacy Through the Use of Pen-Based Computing," International Journal of Engineering Education, vol. 27, no.5, pp. 958-967, 2011.

[14] T. J. Moore, R. L. Miller, R. A. Lesh, M. S. Stohlmann, and Y. R. Kim, "Modeling in Engineering: The Role of Representational Fluency in Students' Conceptual Understanding," Journal of Engineering Education, vol. 102, no. 1, pp. 141-178, 2013. http://dx.doi.org/10.1002/jee.20004

[15] J. H. Joseph, and F. M. Dwyer, "The Effects of Prior Knowledge, Presentation Mode, and Visual Realism on Student Achievement," Journal of Experimental Education, vol. 52, no. 2, pp. 110-121, 1984.

[16] M. J. Nathan, W. Kintsch, and E. Young, "A Theory of Algebra Word Problem Comprehension and Its Implications for the Design of Computer Learning Environments," Cognition and Instruction, vol. 9, no. 4, pp. 329-389, 1992. http://dx.doi.org/10.1207/ s1532690xci0904_2 
[17] G. Padmanabhan, Fluid Mechanics Lab Manual for Civil Engineering Students. Debuque, IA: Kendall/Hunt Publishing Company, 2007.

[18] USEPA, Slurry Trench Construction for Pollution Mitigation Control. Cincinnati, OH: Municipal Environmental Research Laboratory, Office of Research and Development, EPA-540/2-84$001,1984$.

[19] USEPA, Investigation of Slurry Cutoff Wall Design and Construction Methods for Containing Hazardous Wastes. EPA/600/5287/063, 1987.

[20] M. A. Hashim, S. Mukhopadhyay, J. N. Sahu, and B. Sengupta, "Remediation Technologies for Heavy Metal Contaminated Groundwater," J. Environ. Manage., vol. 92, no. 10, pp.23552388, 2011. http://dx.doi.org/10.1016/j.jenvman.2011.06.009

[21] M. Kasi, J. McEvoy, G. Padmanabhan, and E. Khan, "In situ Groundwater Remediation Using Enricher Reactor-Permeable Reactive Biobarrier," Water Environ. Res., vol. 83, no. 7, pp. 604613, 2011. http://dx.doi.org/10.2175/106143011X129288144444 $\underline{57}$

\section{AUTHORS}

M. Kasi, Ph.D. is a Project Engineer at Moore Engineering, Inc., West Fargo, ND 58078 USA. He is actively involved in teaching at North Dakota State University (NDSU), Fargo, North Dakota. He taught laboratory classes for Fluid Mechanics and Environmental Engineering, and gave numerous lectures on modeling surface water quality, stormwater, and groundwater. He was a Teaching Assistant for the Fluid Mechanics Laboratory Class at NDSU in Spring and Fall of 2010 when this
Flownet course module was introduced (e-mail: mkasi@mooreengineeringinc.com).

Y. Chi is a Graduate Engineer at Moore Engineering, Inc., West Fargo, ND 58078 USA. She was a Teaching Assistant in the Fluid Mechanics laboratory for undergraduates at North Dakota State University (NDSU), Fargo, North Dakota in Spring and Fall of 2011. She obtained her Bachelor's degree in Management and Planning of Resources and Urban Area from Anhui University of Science and Technology, China, Master's in Hydrology and Water Resources from China University of Geosciences, China, and a Master's in Civil Engineering from NDSU (e-mail: ychi@mooreengineeringinc.com).

G. Padmanabhan , Ph. D., P.E., M. ASEE, F. ASCE is a Professor of Civil Engineering at North Dakota State University, Fargo, ND 58105 USA. He is a long standing member of ASEE and ASCE. He has taught Fluid Mechanics and associated laboratory courses for several years in the past. He continues to teach Applied Hydrology, Open Channel Flow, and Groundwater courses. He is the Director of the North Dakota Water Resources Research Institute. He has been active in Science, Technology, Engineering and Mathematics (STEM) education outreach activities to minorities at the college and as high and middle school level for the last fifteen years (e-mail: g.padmanabhan@ndsu.edu).

Submitted 25 July 2013. Published as re-submitted by the authors 11 October 2013. 